Karl Lehmann: Die Wirkung hoher Sauerstoffdrücke auf thier. Gebilde. 421

heben, dass ich mich niemals als Anwalt der Localisationslehre gefühlt habe, dass, als ich meine Untersuehungen über diesen Gegenstand begann, es mir ganz gleichgiiltig war, ob die Resultate derselben die eine oder die andere Lehre unterstiitzen werden, und dass es überhaupt ein Lehrsatz der deutschen Naturforschung ist, nicht für eine irgendwie aufgestellte Anschauung Gründe auf Gründe zu häufen, denn da würde der Fleiss, nicht die Wahrheit den Ausschlag geben - sondern eine correcte Fragestellung und zuverlässige Methoden auszusinnen, und die Beantwortung der Frage der Natur selbst zu überlassen. Uebrigens ist die Thatsache von der Rindenlocalisation ganz unabhängig von der Lehre dass „die Centren " nicht ineinanderliegen dürfen, oder dass dieselben eine scharfe Begränzung haben müssen.

(Aus dem physiologischen Laboratorium in Zürich.)

\title{
Die Wirkung hoher Sauerstoffdrücke auf thierische Gebilde.
}

Von

\section{Karl Iehmann,}

med. pract., Assistent am physiologischen Laboratorium in Zïrich.

In seinem Werke: "La pression barometrique, Paris $1878^{\text {" }}$ hat Paul Bert den schädlichen Einfluss hohen Luftdrucks auf eine giftige Wirkung des comprimirten Sauerstoffs zurückgefihrt. Seine Versuche erstrecken sich über das ganze Thierreich, namentlich sind aber Vögel und Hunde von ihm berücksichtigt worden. Ueberall zeigte sich, dass eine Erhöhung des Sauerstoffpartiar-

tionen in den Gehirnhemisphären auftritt, so ist doch die Unterstützung, welche dieser Lehre durch ihn zu Theil wird, von höchst zweifelhaftem Werthe. 
drucks auf mehr als 3 Atmosphären (entsprechend z. B. einem Luftdruck von 15 Atmosphären) in kurzer Zeit das Leben des Thieres unter Convulsionen, Sinken des Blutdrucks, der Temperatur, der Koblensäure- und Harnstoffausscheidung gefährdet. Bert fand die Ursache aller dieser Störungen in einer geringen Steigerung des Sauerstoffgehaltes des Blutes, entsprechend einer enormen Vermehrung der Sanerstoffspannung in demselben. Er kommt bei der Prïfung der Symptome der "Sanerstoff-Vergiftung“ zu dem Schlusse, dass sie seien „le résultat d'une surexcitation des centres nerveux" ähnlich wie bei der Strychnin- und Phenolvergiftung; daneben räumt er aber ein, dass zngleich mit dem Nervensystem auch die andern resistenteren Theile des Organismus leiden.

Zur Erhärtung dieser Annahme hat Bert nur äusserst wenig Material beigebracht: er fand in den 3 in dieser Richtring angestellten Versuchen, dass jedesmal Nerv und Muskeln eines uncomprimirten Froschschenkels länger lebten als die der comprimirten Seite. Näher gehe ich auf diese Versuche, die ich nicht nachuntersucht habe, nicht ein.

Am ausgeschnittenen Herzen hat Bert nur einmal experimentirt, indem er 4 Froschherzen in Humor vitreus vom Hunde brachte und 2 davon mit 10 Atmosphären „d'un air suroxygéné" comprimirte. Nach 2 Stunden findet er die comprimirten: complétement arrêtés, ils ne peuvent pas être rappelés parl'excitation. Die uncomprimirten: Les coeurs présentent encore des battements, surtout pour les oreillettes, on peut les exciter.

Ich komme später auf diesen Versuch zurïck.

Mein verehrter Lebrer, Herr Professor Hermann, beschäftigte sich während der Sommerferien 1881 mit einer Anzahl Versuche, die die kritische Prüfung von Bert's Angaben zum Zwecke hatten. Er arbeitete dabei mit einem unten näher zu beschreibenden kleinen Apparate, der die Anwendung sehr hoher Sanerstoffspannungen bei gleichzeitiger vollkommener Sichtbarkeit des Objects gestattete. Die allerdings nicht sehr zahlreichen Versuche, durchweg mit reinem Sanerstoff von 12-24 Atmosphären angestellt, ergaben ihm in mehreren Fällen ein unerwartet langes Leben kleiner Thiere (Eidechsen, Frösche, Mäuse, Insecten) gegenïber den Bert'schen Angaben, sowie namentlich ein auffallendes Verhalten von Elementarorganen. So blieb Erregbarkeit und Muskelstrom comprimirter Nervenmuskelpräparate Stunden lang constant, 
ebenso der Froschhautstrom, das ausgeschnittene Froschherz pulsirte einige Stunden lang bei sehr starkem Drucke, obwobl es bei seiner Kleinheit sofort dureh und durch von dem gewaltigen Sauerstoffdruck imprägnirt sein musste.

Diese vorläufigen Resultate schienen eine ansgedehntere Prüfung der ganzen Frage nach dem Grade und der Art der Schädlichkeit des comprimirten Sauerstoffs wünschenswerth zu machen, eine Untersuchung, die mir Herr Professor Hermann, den andere Arbeiten von der Verfolgung dieser Frage abhielten, zu übertragen die Guite hatte. Bei derselben stand mir Herr Professor Hermann stets auf das liebenswïrdigste mit seinem Rathe bei, wofür ich ihm meinen besten Dank sage.

Meine Untersuchung beschränkte sich bisher vorwiegend darauf, das Verhalten des ausgeschnittenen Froschherzens in sorgfältig gereinigtem, comprimirtem Sauerstoff und Stickstoff zn beobachten, indem ich es mir fast stets angelegen sein liess, Controlversuche mit Herzen in gewöhnlicher Luft anzustellen.

Ehe ich meine eigenen Versuche darstelle, möchte ich noch kurz die wichtigsten Angaben Bert's über das Verhalten des Herzens im comprimirten Thier erwähnen.

5 Frösche, die einzeln einem Sauerstoffpartiardruck von $21 / 8$ -5 Atmosphären ausgesetzt wurden, waren nach 19-43 Stunden reflexlos, schienen todt. Bei vieren derselben schlugen die Herzen beim Oeffnen der Brusthöhle noch. In einem Versuche war das Herz in situ belassen, aber blossgelegt, nach $18^{1 / 2}$ Stunden waren die Ventrikelcontractionen irregulär und spärlich geworden, die Herzohren contrahirten sich dagegen häufig (40 pro Minute), ein Verhalten wie ich es auch öfters sab.

An den Hunden war bei einer Sauerstoffspannung von 4-6 Atmosphären meist eine auffallende Herzverlangsamung $\mathrm{zu}$ beobachten, genauere Angaben fehlen. Das Herz zeigte sich meist als das "ultimum moriens" an den unter Convulsionen und Lähmungen sterbenden Thieren.

Diese Angaben liessen von vorne herein das Herz als ein gegen Sauerstoffcompression resistentes und desshalb für meine Versuche günstiges Organ erseheinen, sodann kam aus oben erwähnten Gründen die Kleinheit und vor allem die automatische, rhythmische, leicht sichtbare Bewegung für die Wahl dieses Objectes in Betracht. 
Für meine Versuche standen mir folgende Apparate zu Gebote: Eine Natterer'sche Compressionspumpe mit 2 zugehörigen schmiedeisernen, etwa 1 Liter fassenden Recipienten, wie sie zur Darstellung der flüssigen Kohlensäure gebraucht werden. In diesen Recipienten bewahrte ich meine gewaschenen, comprimirten Gase auf, an sie wurde eine kleine von Professor Hermann construirte Gaskammer angeschraubt und aus ihnen gefuillt.

Diese Gaskammer besteht aus einem Glaseylinder von 5 Millimeter Dicke, 73 Millimeter Höhe und 40 Millimeter lichter Weite (Volum 90 Cubikcentimeter), der oben und unten durch eine mit einer Nathe and Lederring versehene Messingplatte geschlossen wird. Der feste Verschluss geschieht dadurch, dass 3 in die obere Messingplatte eingesetzte Messingsäulen durch entsprechende Oeffnungen der unteren Platte durchgesteckt werden, die an ihrem unteren Ende ein Gewinde tragen, in das sehr stark anziehbare Schraubenmuttern passen. Die obere Platte ist mit einer durchbohrten durch einen Hahn verschlossenen Säule versehen, durch welche das Gas eingelassen wird, indem das obere Sänlenende an den eisernen Recipienten angeschraubt wird. Zugleich trägt die obere Platte ein Capillarmanometer mit Quecksilbertropfen, häufig wurde auch die Auffangung des gebrauchten Gases in calibrirten mit Wasser gefüllten Gefässen zur Bestimmung des Druckes angewendet.

Die untere Messingplatte wird durch zwei isolirte Kupferdrähte durchbohrt, die an ihren beiden Enden mit Klemmschrauben versehen sind, um sowohl Ströme aus dem Apparate ableiten, als um darin befindlichen Organen solche zuleiten zu können.

Der sehr handliche Apparat gestattet eine vorziigliche Uebersicht der darin vorgehenden Dinge, er hat Driicke bis zu 30 Atmosphären ausgehalten; doch ist mir auch einmal ein Unfall passirt, den ich zur Warnung glaube mittheilen zu milssen. Der Glascylinder, der einen ganz feinen, kurzen, oberflächlich scheinenden Sprung davongetragen, aber seitdem wieder mehrere Compressionen gut ausgehalten hatte, explodirte plötzlich auf meinem Arbeitstische, ohne dass eine Viertelstunde lang irgend Jemand in Zimmer gewesen wäre. Der Apparat hatte 20 Atmosphären Sauerstoff enthalten, der Glascylinder war in unzählige Splitter geborsten, die z. Th. in Papier and Holz in beträchtlichen Entfernungen eingedrungen waren. Seitdem arbeite ich nur noch mit ganz intacten Glascylindern, die auf das sorgfältigste behandelt werden. 
Der Apparat wurde stets unter Wasser aufbewahrt, weil dies die Erhaltung einer viel constanteren Temperatur gestattete als die Aufbewahrung in Luft, und zugleich den Vortheil bot, jedes Entweichen von Gasblasen sichtbar zu machen. Es wurden zur Publication nur vollständig gelungene Versuche verwendet, eine grosse Anzahl, bei denen entweder der Apparat nicht schloss, oder die aus sonst einem Grund nicht einwandfrei waren, wurden von der Berücksichtigung ausgeschlossen.

Im Apparate war stets reichlich für Feuchtigkeit gesorgt durch feuchte Fliesspapierstreifen; in 2 Versuchen, bei denen ich die sich bildende $\mathrm{CO}_{2}$ ausscbliessen wollte und desshalb einen kleinen Tiegel mit Kali-Stuicken anbrachte, wurden noch reichlich feuchte Schwämmchen beigegeben. Es erwies sich diese Modification uibrigens, wie erwartet, als vollkommen gleichgültig für den Verlauf des Versuchs.

Die Herzen wurden anfangs mit dem Bruststïek des Froschs in Verbindung gelassen, und 2 solche Bruststücke mit dem Rücken gegeneinander an einem vertikalen Glasstreifen befestigt; die so erhaltenen Resultate waren mir aber nicht constant genug, und ich wählte daher für alle publicirten Versuche folgende Methode: Es wurden je 3 Herzen zusammen verwendet. Jedes wurde mit Schonung des Venensinus nach Spaltung des Herzbentels herauspräparirt, indem dasselbe an den durchschnittenen Aorten gehalten wurde. Die herausgenommenen Herzen wurden, stets mit dem Venensinus nach oben (nach Bezold, Virchow's Archiv 14. pag. 228) neben einander auf den Deckel eines Porcellantiegels gelegt, das Blut, das sie auspressten, mit einer feinen Pipette abgesogen und erst, wenn sie nöthigenfalls durch Umlagerung trocken gelegt waren, wurden sie in den Compressionsapparat gebracht, wo es nur sehr selten noch zur Auspressungen von nennenswerthen Flïssigkeitsmengen kam.

Sowie es im geringsten den Anschein hatte, es sei ein Herz etwas verletzt, sowie seine Schlagfolge nach der Entnahme irgend welche grobe Abnormitäten darbot, wurde es sofort durch ein anderes ersetzt.

Die Pulsfrequenz von 3 nebeneinanderliegenden Herzen war meist eine ziemlich ähnliche, $15-20$ pro $1 / 2$ Minute, unmittelbar nach der Präparation zeigten sich manchmal für kurze Zeit 8-27 Pulse pro $1 / 2$ Minute. Beinahe neben jedem Compressionsversuch wurde 
ein Parallelversuch mit 2-3 in gleicher Weise präparirten Herzen gemacht, die ebenfalls auf einem Tiegeldeckel in einem halb mit feuchtem Sand gefüllten gut verschlossenen Glasgefäss auch unter Wasser versenkt wurden. Es kamen Ranae esculentae und temporariae zur Verwendung ohne bemerkenswerthen Untersehied, und zwar, da die Versuche im November, December und Januar angestellt sind, ausschliesslich Winterfrösche.

Leider zeigen eine ziemliche Anzahl der Versuche den Mangel, nicht bis zu Ende beobachtet worden zu sein, ein Umstand, an dem ich nicht Schuld bin. Die Versuche wurden meist Morgens 10 Uhr nach der Vorlesung, in der ich zu assistiren hatte, begonnen, zwisehen $11^{1 / 2}$ und $1 \mathrm{Uhr}$ war die Compression fertig, und ich beobachtete nun bis Abends 8-9 Uhr, ja bis $10 \mathrm{Uhr}$, was, da unser Laboratorium im Universitätsgebäude liegt, die spätest mögliche Zeit ist.

Der verwendete Sauerstoff wurde ausschliesslich durch Erhitzen von Braunstein und chlorsaurem Kali und Auffangen über Wasser dargestellt.

Anfangs wurde der 0 ungereinigt angewendet, dann aber, als ich den friheren Stillstand der comprimirten gegenüber den Controlherzen fand, wurde er stets durch 2 Waschflaschen mit Natronlauge, in den letzten Versuchen auch noch durch ein U-Robr, das mit Natronkalk und Chlorcalciumstïckehen gefüllt war, geleitet. - Alle diese Reinigungen hatten anf das Resultat der Versuche keinen Einfluss, ich habe desshalb in die folgende Tabelle anch die Versuche mit ungereinigtem Sauerstoff aufgenommen.

Dagegen mischte sich durch das Waschen und Comprimiren stets etwas Stickstoff dem Sauerstoff bei, über dessen Menge ich jedoch keine genauen Ermittelungnn anstellte. Die Zahlen der folgenden Tabelle geben den abgelesenen Sauerstoffdruck ohne Correction an, sind also wohl etwas zll gross; wie die Tabelle zeigt, ist aber der Untersebied der Wirkung von 10 und $16^{1 / 2}$ Atmosphären Sauerstoff auf das Froschherz nicht mehr angebbar, so dass dieser Fehler nichts zu sagen hat.

Die Temperatur des Wassers, in dem die Gaskammer aufbewahrt war, schwankte von $14-16^{\circ}$ Cels., selten kamen Temperaturen von $1-2^{0}$ weniger, sehr selten wenig höhere vor. - Behufs genauer Beobachtung wurde die Gaskammer ab und zu auf Minuten dem Wasser entnommen. 
Die Wirkung hoher Sauerstoffdrücke auf thierische Gebilde.

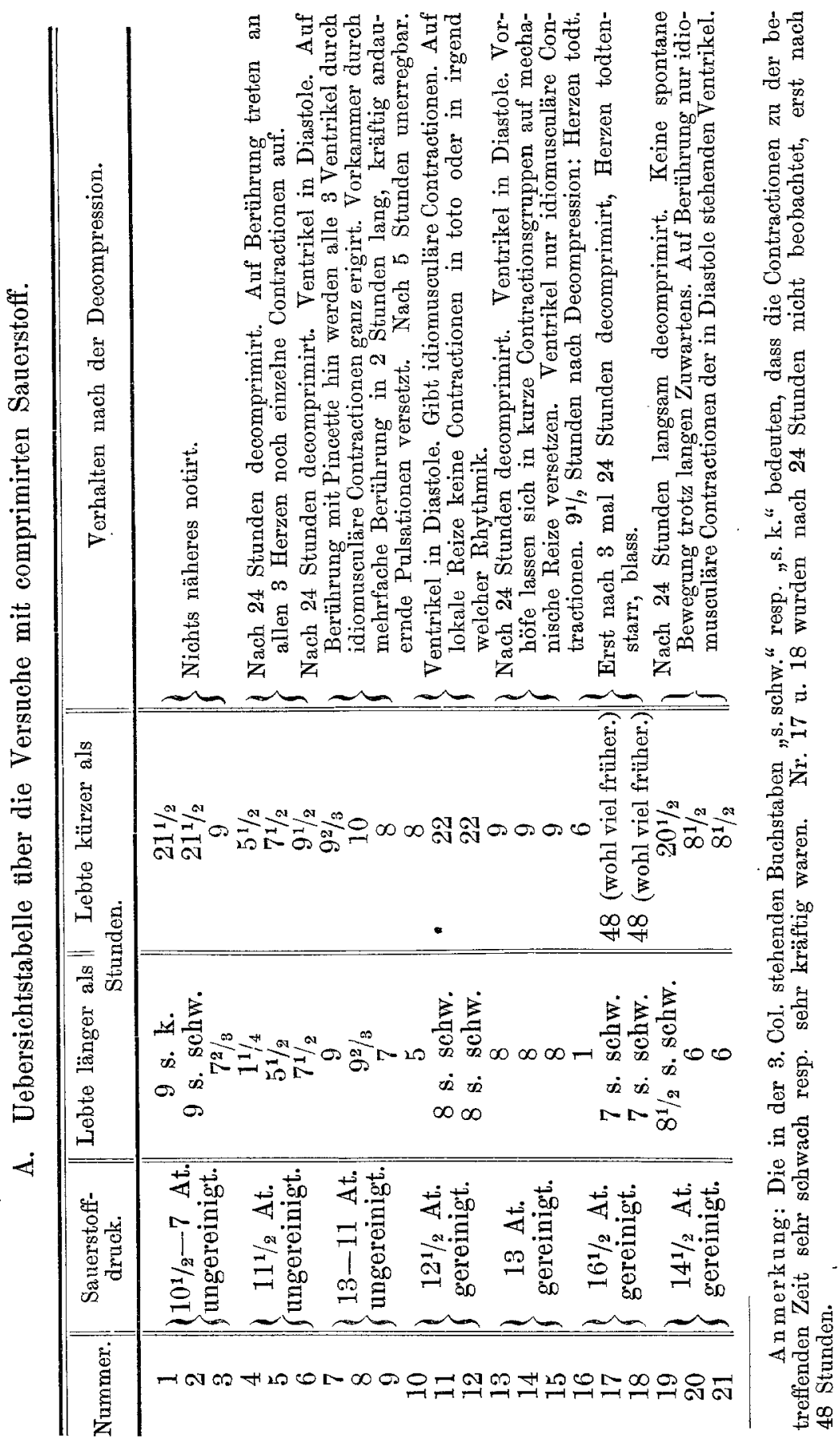


Die Dauer der Contractionen wurde nach der letzten Bewegung:sspur bemessen, die an Venensinus oder Herzohr noch wahrzunehmen war, was jedesmal 3-4 Stunden länger dauerte als das Verschwinden der letzten Kammercontraction. Die Beobachtung der fast erlöschenden Contractionen wurden stets mit grosser Sorgfalt ausgefïhrt und öfters 10-15 Minuten darauf verwendet.

Betrachten wir die vorstehende Tabelle, so fällt uns sofort auf, dass bei keinem einzigen Herzen länger als $9^{2} / 3$ Stunden Contractionen beobachtet sind, dass dagegen um diese Zeit von 22 Herzen 14 stillstanden, während die andern (mit einer einzigen Ausnahme), als die Beobachtung unterbrochen werden musste, nur noch minime, oft sehr schwer sichtbare Contractionen einzelner Vorhofstellen aufwiesen, und kein einziges Herz am andern Morgen d. h. nach 20-22 Siunden in spontaner Contraction gefunden wurde. Ich bin fest davon überzeugt, dass die allergrösste Mehrzahl dieser Abends minim pulsirenden Vorhöfe in 1-2 Stunden auch zur Ruhe gekommen sind, da bei der Compression mit Sauerstoff die Abnahme der Ausdehnung und Intensität der Compressionen, sowie die ersten Stunden vorbei sind, eine sehr rasche ist.

Wir düren also sagen: Nach 10 Stunden circa hat die Mehrzahl der Herzen bei einem Druck von 9-16 Atmosphären Sauerstoff zu schlagen aufgehört, länger als 22 Stunden hat sicher keines geschlagen.

In der letzten Colonne der Tabelle habe ich einige Beobachtungen ïber das Verhalten der Herzen nach der Decompression niedergelegt. Es geht daraus ganz allgemein hervor, dass die in der Diastole befindlichen Herzen nach 24stündiger Compression noch einen Rest von Lebensfähigkeit bewahrt haben, an idiomuskulären, wulstförmigen Contractionen der Ventrikel auf Berührung fehlte es nie, und es gelang häufig grössere oder kleinere Gruppen von Vorhofcontractionen auf Berührung hervorzubringen. -

Ich theile das Protokoll von einem meiner Versuche etwas ausfïbrlicher mit, um daran noch einige Bemerkungen über nebenbei beobachtete Erscheinungen zu knüpfen. 
3 mittlere Esculentae. Herzen präparirt wie oben beschrieben. Die Pulszahlen verstehen sich pro halbe Minute.

\begin{tabular}{|c|c|c|c|c|c|}
\hline Zeit. & Druck. & Nr. 19. & Nr. 20. & $\mathrm{Nr} .21$. & Temp. \\
\hline $\begin{array}{c}12,46 \\
1,- \\
3,- \\
4,- \\
5,- \\
6,30\end{array}$ & $\begin{array}{c}1 / 5 \\
1 / 5 \\
141 / 2\end{array}$ & $\begin{array}{l}16 \text { kräftig. } \\
12-13 \text { kräftig. } \\
13 \text { zieml. kräftig. } \\
\text { Kraft der Ven- } \\
\text { trikel vermind. } \\
\text { idem } \\
\text { Keine deutliche } \\
\text { Ventrikel-Con- } \\
\text { traction mehr. } \\
\text { Vorhof zeigt } \\
\text { schw. Pulsation. } \\
\text { Porhof zeigt } \\
\text { noch spurweise } \\
\text { Contractionen. }\end{array}$ & $\begin{array}{c}13 \text { kräftig. } \\
8 \text { kräftig. } \\
6 \text { kräftig. Vor- } \\
\text { höfe } 2-3 \text { mal } \\
\text { rascher. } \\
8 \text { kräftig. } \\
8 \text { kräftig. } \\
3-4 \text { kräfttig. } \\
\text { Kraft der Ven- } \\
\text { trikel vermind. } \\
\text { idem. } \\
\text { Ventrikel kräft. } \\
\text { 3-4. Vorhof } \\
\text { ziemlich öfter. } \\
\\
\text { Ruhe. }\end{array}$ & $\begin{array}{l}13 \text { kräftig. } \\
14 \text { kräftig. } \\
\text { 18-19 kräftig. } \\
\\
16 \text { kräftig. } \\
16 \text { kräftig. } \\
13 \text { kräftig. } \\
\text { Kraft der Ven- } \\
\text { trikel vermind. } \\
\text { idem. } \\
\text { Ventrikel } 3-4 \\
\text { ziemlich kräftig. } \\
\text { Vorhof ziemlich } \\
\text { öfter. } \\
\text { Ruhe. }\end{array}$ & $15^{1} /_{2}^{0}$ \\
\hline $\begin{array}{r}\text { 7. I. Morg, } \\
8,30 \\
10,-\end{array}$ & $"$ & $\begin{array}{c}\text { Ruhe. } \\
\text {, }\end{array}$ & $"$ & $"$ & \\
\hline
\end{tabular}

Darauf Decompression.

Wir haben in diesem Beispiel in N. 19 und N. 21 den gewöhnlichen Verlauf eines Compressionsversuchs. Durch die Compression wird für $1 / 4-1 / 2$ Stunde gewöhnlich (zuweilen noch für länger) die kräftige Intensität der Contraction zu einem Maximum gesteigert, dabei ist (wie hier) häufig die Frequenz vorübergehend etwas vermehrt, doch kann diese Vermehrung auch fehlen, ja sogar eine kleine Verlangsamung habe ich ab und zu beobachtet, doch achtete ich nicht in allen Fällen genauer auf die Frequenz.

Nach 4 Stunden fängt in obigen beiden Versuchen wie gewöhnlich die Ventrikelthätigkeit, die bis dahin ziemlich unverändert war, an, sich zu verlangsamen, zu erlahmen, im Laufe der nächsten 1-2 Stunden kommt es zum Ventrikelstillstand (oft noch friber) und nun schlagen die Vorhöfe noch einige Stunden fort. - Der Versuch N. 20 bietet mit seiner geringen Anzahl Contractionen einen Fall dar, wie er nicht häufig beobachtet wurde.

Ich komme zu den Controlversuchen an uncomprimirten Herzen in Luft. 
Die Resultate derselben ${ }^{1}$ ) sind untereinander, ganz wenige Ausnahmen abgerechnet, eben so tibereinstimmend wie die der Versuche mit comprimirtem Sauerstoff unter sich, da die Frösche, die dazu verwendet wurden, aus derselben Quelle bezogen waren, die Temperatur bei allen Versuchen etwa die gleiche $14-16^{0}$ (seltene Extreme 12 und $1^{\circ}$ ), and ebenso die Präparationsweise ganz die gleiche blieb.

$\mathrm{Zu}$ der folgenden Tabelle bemerke ich, dass eine Anzahl Versuche nach 2 mal 24 Stunden und mehr abgebrochen wurde, weil mir diese Leistung vollkommen genügte im Vergleich mit der der comprimirten Herzen.

B. Uebersichtstabelle üb er die Controlversuche in Luft zu denen in comprimirtem Sauerstoff.

\begin{tabular}{|c|c|c|c|}
\hline $\mathrm{Nr}$. & $\begin{array}{c}\text { Controlversuche zu Nr. } \\
\text { der Tabelle A. }\end{array}$ & $\begin{array}{c}\text { lebte länger } \\
\text { als } \\
\end{array}$ & len. \\
\hline $\begin{array}{r}1 \\
2 \\
3 \\
4 \\
5 \\
6 \\
7 \\
8 \\
9 \\
10 \\
11 \\
12 \\
13 \\
14 \\
15\end{array}$ & 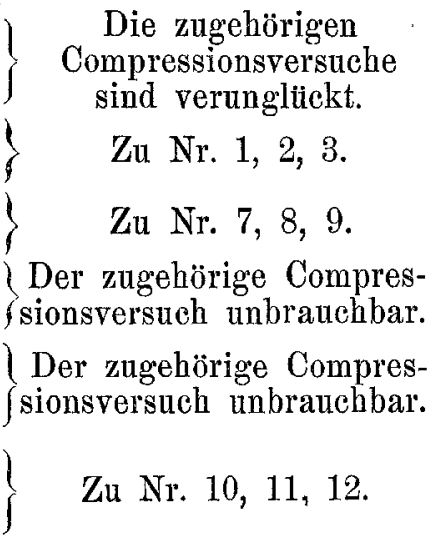 & $\begin{array}{l}24 \\
28 \\
81 / 2 \\
48 \\
48 \\
10 \\
22 \\
48 \\
48 \\
36 \\
24 \\
36 \\
46 \\
56 \\
56\end{array}$ & $\begin{array}{c}48 \\
\text { unbeobachtet. } \\
31^{1 / 2} \\
\text { unterbrochen. } \\
\text { unterbrochen. } \\
22 \\
24 \\
\text { unterbrochen. } \\
\text { unterbrochen. } \\
46 \\
36 \\
46 \\
56 \\
\text { unterbrochen. } \\
\text { unterbrochen. }\end{array}$ \\
\hline
\end{tabular}

1) Ich bin oben nicht auf das Eingangs erwähnte Experiment Bert's am ausgeschnittenen Froschberzen eingegangen, weil seine Controlherzen, die nach 2 Stunden schon eine starke Schädigung zeigten, mir deutlich gegen die Zulässigkeit der Aufbewahrung des Herzens im Humor aqueus des Hundes zu sprechen schienen.

Auch die Castell'schen Versuche über das Froschherz in verschiedenen Gasen (Archiv f. Anat. u. Physiolog., 1854, p. 226) sind für Vergleiche nicht verwendbar, da er das Herz unter normalem Drucke bei Zimmertemperatur in Luft nur ca. 3 Stunden schlagend fand, also jedenfalls wie auch schon 
Aus dieser Tabelle folgt sofort, dass bei $14-16^{\circ}$ das ausgeschnittene Froschherz meist uiber 24, öfters über 48, ja bis 56 Stunden schlägt, und dass ein Absterben nach 8-10 Stunden, wie es für Herzen unter starker Sauerstoffcompression die Regel ist, hier eine ganz seltene Ausnabme bildet, bei der man immel unwillkürlich daran denkt, ob das betreffende Herz auch nicht etwa verletzt war ete. Allerdings standen die Ventrikel oft ziemlich parallel mit denen in comprimirtem Sauerstoff still, und die längere Lebensdauer betraf namentlich die Vorböfe.

Es folgt also ohne weiteres, dass die Compression mit Sauerstoff eine entschiedene Schädigung ist, anf deren Würdigung ich aber erst nach Erwähnung des 3. Theiles meiner Versuche eingehen will.

Um zu ermitteln, ein wie grosser Theil der Schädlichkeit des comprimirten Sauerstoffs auf Rechnung des Drucks an sich komme, lag es sehr nahe Versuche mit dem indifferenten Stickstoff zu machen, dem man soviel Sauerstoff beimischte, dass die normale Sauerstoffspannung von $1 / 5$ Atmosphäre neben einer möglichst hohen Stickstoffspannung erreicht würde. Es galt also möglichst reinen Stickstoff in meinen mit gewöhnlicher Luft gefüllten Glasrecipienten aus dem Eisenrecipienten einströmen zu lassen.

Den $\mathrm{N}$ stellte ich auf den freundlichen Rath von Herrn Prof. Victor Meyer in folgender Weise dar:

1 Theil Ammoniumnitrat,

1 Theil käufliches Natriumnitrit,

1 Theil doppeltehromsaures Kali

werden mit 3 Theilen Wasser vorsichtig erwärmt, da die Reaction, wenn sie einmal eingeleitet ist, sehr stürmisch eintritt. Etwas mehr Wasserzusatz vermindert die Heftigkeit der Reaction, scheint aber auch die Menge des gebildeten Stickstoffs zu vermindern.

Das so erhaltene Gas wurde durch 1 Flasche mit Natronlauge, eine mit Schwefelsäure und durch das oben erwähnte $\mathbf{U}$ Rohr in ein zweites Gasometer geleitet.

In 2 Untersuchungen stellte sich heraus, dass der Inhalt des Eisencylinders etwa 2-3\% Sauerstoff enthalte, eine Sauerstoff-

Bernstein nachwies, eine Anzahl wichtiger Vorsichtsmassregeln ausser Acht liess. - Ganz gut stimmen dagegen meine Erfahrungen zu denen, die A ubert von Valentin und sich in Hermann's Handbuch darlegt, freilich indem er sie als durch besonders günstige Umstände bedingt darstellt. 
Karl Lehmann:

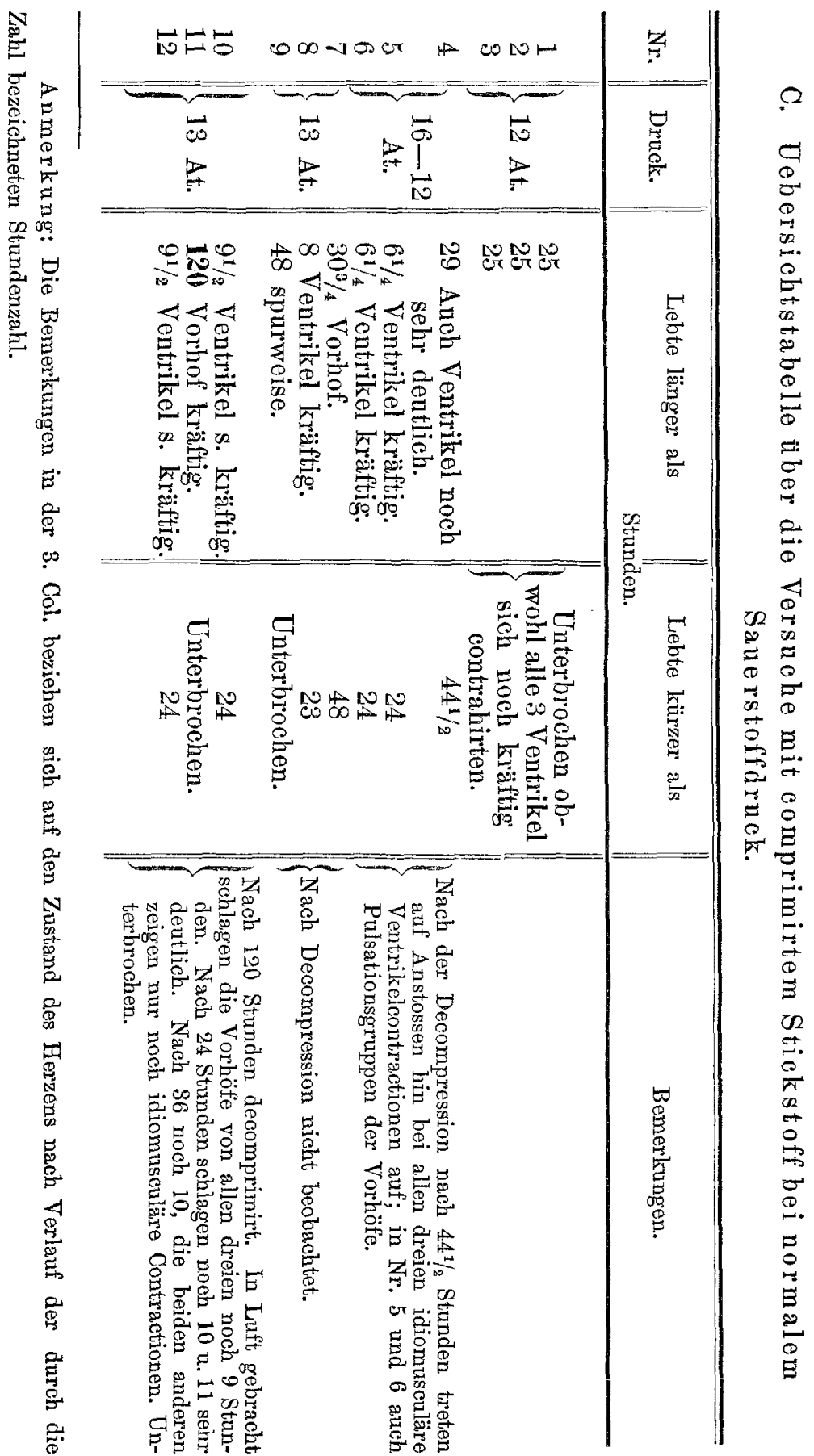


menge, die für meine Zwecke gleichgültig ist, da eine Erhöhung des Stickstoffpartiardrucks auf 15 Atmosphären die Sauerstoffspannung höchstens auf $45 / 100$ Atmosphären bringt, wozu noch die 1/s Atm. Kommt, die der Glascylinder vor der Compression enthielt. Dies schien vernachlässigt werden zu dürfen, und der Erfolg zeigte denn auch, dass von einer bedeutenden Schädigung nicht wohl die Rede sein kann. Noch mehr als bei den Sauerstoffversuchen störte bei diesen Versuchen, dass das zu Ende Beobachten auf sehr grosse Schwierigkeiten stiess, indem häufig Abends die Herzen sehr kräftig schlugen und am Morgen regungslos gefunden wurden.

Von 16 Herzen schlugen bei allen nach 6-10 Stunden die Ventrikel noch kräftig, bei 7 Herzen dauerte das Pulsiren länger als 24 Stunden, zn welcher Zeit auch die Ventrikel noch häufig: in lebhafter Thätigkeit waren. Ich muss zugeben, dass diese Versuchsresultate noch nicht so übereinstimmend sind, wie sie es wohl sein könnten, doch schien es mir namentlich auf diejenigen Versuche anzukommen, die eine lang e Lebensdauer bewiesen, Versuche, wie ich bei der Compression mit Sauerstoff keinen einzigen zu verzeichnen hatte. Es zeigt sich regelmässig nach der Compression die Intensität der Contractionen auf ein Maximum gesteigert (ganz ähnlich wie bei der Sauerstoffcompression), auch hier lässt sich zuweilen eine geringe Beschleunigung beobachten.

In den Versuchen, die nach 24 Stunden schon zu Stillstand geführt haben, füllt als charakteristisch das ausnahmslose, kräftige Schlagen der Ventrikel zu einer Zeit auf, wo die mit Sanerstoff comprimirten Herzen höchstens noch Contractionsspuren der Vorhöfe aufweisen.

Wenden wir uns nun zum Schlusse zu einem Ueberblick unserer Resultate, so zeigt sich die Compression mit Sauerstoff als eine ziemlich beträchtliche, die mit Stickstoff (bei normalem Sanerstoffdruck) theils als eine geringe, theils als gar keine Schädlichkeit.

Die nachtheilige Wirkung des comprimirten Sauerstoffs kann also nicht der mechanische Effect des Drucks sein.

Bedenkt man nun, dass bei der Kleinheit des Organs jedes Theilchen sofort der vollen Wirkung des hochgespannten Sauerstoffs ansgesetzt sein muss, die Lebensprocesse aber stundenlang fast ungestört fortdauern, so kommt man zu der Vorstellung, dass 
die nachgewiesene Schädlichkeit der hohen Sanerstofftension nicht den Character eines unmittelbaren Giftes hat.

Auf eine befriedigendere Erklärung schien folgender Gedankengang zu führen: Nach den neueren Anschauungen ïber das Wesen der Lebensvorgänge, wie wir sie namentlich Hermann und Pflüge r verdanken, finden in der lebenden Zelle Spaltungsprocesse statt, die (namentlich beim Kaltblüter) lange Zeit ohne Sauerstoffzufuhr fortdauern können. Schliesslich hören sie aber auf, wenn nicht durch das Hinzutreten von Sauerstoff anf dem Wege der oxydativen Synthese neues spaltungsfähiges Material gebildet wird.

Es wäre nun möglich, dass im comprimirten Sauerstoff das Uhrwerk, das im Herzen aufgezogen ist, zwar ungestört ablaufen könnte, dass aber ein neues Aufziehen desselben unterbleiben müsste, mit einem Worte, dass comprimirter Sanerstoff vom Herzen nicht oder nur in sehr geringem Maasse zur Restitution seiner spaltungsfähigen, spannkraftfiihrenden Substanzen verwendet werden könnte.

Nicht iibel stimmen die von mir am Froschherz gefundenen Resultate zu den Ergebnissen der Untersuchung von van Overbeek de Meijer ("Over den invloed van zuurstoffgas onder hoogere drukking op lagere organismen en levende grondformen." - Onderzoekingen gedaan in het physiologische laboratorium der Utrechtsche hoogeschool. Derde reeks VI. Utreeht 1881.)

$\mathrm{Er}$ untersuchte namentlich Flimmerhaare von verschiedenen Thieren in der Donders'schen Gaskammer und fand, dass Sauerstoffdruck von 8 Atmosphären die Flimmerbewegung der Austerkiemen rasch zum Stillstand bringt, dass nach der Decompression die Bewegungen aber rasch wiederkelren; andere Flimmerhaare, zum Beispiel von der Süsswassermuschel, hielten eine Compression mit 10-12 Atm. Sauerstoff mehrere Stunden ohne Nachtheil aus, während das Flimmerepithel des Froschrachens sich viel empfindlicher gegen Compression zeigte. (Vergl. eine gegentheilige Angabe Tarchan off's bei Engelmann in Hermann's Handb. I., p. 400.) Achnliche Resultate erhielt er an Spermatozoen, Infusorien, jungen Barschen ete., wobei bald eine etwas grössere bald etwas geringere Resistenz gefunden wurde. Am widerstandsfähigsten blieben Bakterien, die selbst nach zweimal 24 Stunden bei 12 Atmosphären theilweise noch Bewegungen zeigten.

Am ausgeschnittenen Froschherzen scheint van Orerbeek de Meijer uur einen oder wenige Versuche gemacht zu haben und 
zwar von einem anderen Gesichtspunkt aus als ich, er beobachtete nicht die Lebensdauer, sondern die Pulsfreonenz in erster Linie, die er [in dem einzigen und zwar noch dazu sehr kurz von ihm erwähnten Versuche] nach einer geringen anfänglichen Zunahme bei 10 Atmosphären Sauerstoff nach 41 Minuten plötzlich auf die Hälfte der ursprünglichen Zahl sinken sah; mehrmaliges Erhöhen und Vermindern der Spannung erhöhte auch wieder die Schlagfolge auf das normale, oder setzte sie wieder von neuem auf die Hälfte herab.

Van Overbeek de Meijer kommt durch seine Erfahrungen zu ähnlichen Schlïssen wie ich, auch ihm ist der comprimirte Sauerstoff eine Schädlichkeit zweiten Rangs, die lange Zeit das Leben latent macht, ehe sie es vernichtet. Ganz anders verhält sich, wie der Autor an einer Reihe der von ihm auch in comprimirtem Sauerstoff untersuchten Gebilde zeigt, das Wasserstoffhyperoxyd, das selbst in sehr schwachen Lösungen ein Gift ist, dessen Wirkung weit die Scbädlichkeit des comprimirten Sauerstoffs hinter sich lässt (entgegen den Angaben von Regnard und Guttmann, die die Wirkung des comprimirten Sauerstoffs auf die von Wasserstoffhyperoxyd zurïckzuführen versuchten).

Auf die Polemik van Overbeek's gegen Bert trete ich nicht ein und die von dem holländisehen Forscher allerdings nur als bescheidene Vermuthung gegebene Erklärung: „Der comprimirte Sauerstoff wirke dadurch schädlich, dass er mit dem Protoplasma eine undissociirbare, also für das Leben nutzlose Verbindung bilde," erwähne ich auch nur, ohne auf eine Kritik derselben einzugehen, ebe ich für meine Vermuthung mehr Beweise beibringen kann.

Pflüger hat in seiner bekannten Arbeit: „Die physiologische Verbrennung in den lebenden Organismen" (Pflügers Archiv Band X.) namentlich auf das Resultat Bert's Gewicht gelegt, dass die Stoffwechselproducte $\left(\mathrm{CO}_{2}\right.$ und Harnstoff $)$ bei den Thieren im comprimirten Sauerstoff vermindert gefunden wurden. Er stellt darauf die Vermuthung auf: der Tod trete ein, "weil die Zelle die Fähigkeit sich Sauerstoff zu nehmen, verloren habe." Da Pflüger aber grade in dieser Arbeit die Rolle des Sauerstoffs als Restitutionsmittel so sehr betont hat, so glaube ich, diesen Ausspruch so deuten zu dürfen, dass auch $P$ flüger an verhinderte Restitutionsprocesse gedacht habe, wodurch allerdings erst mi ttelbar die Stoffwechselproducte vermindert würden. 
Pflüger's sinnreicher Vergleich der comprimirten Zelle mit dem Phosphor, der in reinem Sauerstoff nicht leuchtet, veranlasste mich, über diesen letzteren Gegenstand eine Reihe ron bis jetzt noch nicht abgeschlossenen Versuchen zu machen, über welche ich glejchzeitig mit einer Serie nener Experimente über das Frosehherz in Kürze hoffe berichten zu können.

Ich hätte wohl auch mit der Publication des hier vorliegenden seit 8 Wochen vollendeten ersten Theils meiner Arbeit noch gewartet, wenn mir nicht Herr Professor Hermann einen ihm soeben zugegangenen Separatabdruck einer vorläufigen Mittheilung von E. de Cyon an die Pariser Academie (datirt vom 20. Februar 1882) mitgetheilt hätte, worin sich dieser Forscher nach unter schwachem Druck (bis 3 Atmosphären Sauerstoff) an Hunden gemachten Versuchen zu dem Schlusse für berechtigt hält:

"L'oxygène n'est nullement un poison spécial pour l'organisme."

\section{Ueber subjective Schwankungen der Intensität acustischer Empfindungen.}

Von

\section{Victor Vrhantschitsch}

in Wien.

Bei meinen Hörversuchen fiel es mir auf, dass die Intensität der Gehörsempfindungen bei den meisten Versuchsindividuen stete Schwankungen erkennen lässt, die zuweilen nur unbedeutend häufig, jedoch sehr beträchtlich erscheinen.

Derartige Schwankungen treten sowohl beim monotischen als beim binotischen Hören, beim letzteren jedoch viel auffälliger hervor.

Auf einer ungleichen Perzeptionsfähigkeit des Hörorganes beruht wohl die von mir bereits bei Besprechung des Abklingens acu- 